\title{
PARTIAL DETERMINANT INEQUALITIES FOR POSITIVE SEMIDEFINITE BLOCK MATRICES
}

\author{
Yongtao Li, XiQin Lin And LiHUA FEnG*
}

Abstract. We present some inequalities related to partial determinants for positive semidefinite block matrices. Firstly, we introduce the definition of partial matrix functions corresponding to partial traces and partial determinants, and then we provide a unified extension of a recent result of Lin [10], Chang-Paksoy-Zhang [4] and Lin-Sra [12]. Secondly, we give a new generalization of a result of Paksoy-Turkmen-Zhang [15]. Finally, we conclude with an interesting conjecture involving partial determinants.

Mathematics subject classification (2020): 15A45, 15A60, 47B65.

Keywords and phrases: Block matrices, generalized matrix function, partial traces, partial determinants.

\section{REFERENCES}

[1] R. Bhatia, Matrix Analysis, GTM 169, Springer-Verlag, New York, 1997.

[2] R. Bhatia, Positive Definite Matrices, Princeton University Press, Princeton, 2007.

[3] W. Berndt, S. SRA, Hlawka-Popoviciu inequalities on positive definite tensors, Linear Algebra Appl. 486 (2015) 317-327.

[4] H. Chang, V. E. PAKsoy, F. ZHANG, An inequality for tensor product of positive operators and its applications, Linear Algebra Appl. 498 (2016) 99-105.

[5] D. CHOI, Inequalities related to trace and determinant of positive semidefinite block matrices, Linear Algebra Appl. 532 (2017) 1-7.

[6] D. J. HARTFIEL, An extension of Haynsworth's determinant inequality, Proc. Amer. Math. Soc. 41 (1973) 463-465.

[7] E. V. Haynsworth, Applications of an inequality for the Schur complemment, Proc. Amer. Math. Soc. 24 (1970) 512-516.

[8] R. A. Horn, C. R. Johnson, Matrix Analysis, 2nd ed., Cambridge University Press, Cambridge, 2013.

[9] Y. Li, L. FENG, Extensions of Brunn-Minkowski's inequality to multiple matrices, Linear Algebra Appl. 603 (2020) 91-100.

[10] M. LIN, A determinantal inequality for positive definite matrices, Electron. J. Linear Algebra 27 (2014) 821-826.

[11] M. LiN, An Oppenheim type inequality for a block Hadamard product, Linear Algebra Appl. 452 (2014) 1-6.

[12] M. LIN, S. SRA, A proof of Thompson's determinantal inequality, Math. Notes 99 (2016) 164-165.

[13] Y. MAO, Extensions of Hartfiel's inequality to multiple matrices, Linear Algebra Appl. 589 (2020) 96-102.

[14] R. MERris, Multilinear Algebra, Gordon \& Breach, Amsterdam, 1997.

[15] V. PAKsoy, R. TURKMEN, F. ZHANG, Inequalities of generalized matrix functions via tensor products, Electron. J. Linear Algebra 27 (2014) 332-341.

[16] D. PetZ, Quantum Information Theory and Quantum Statistics. Theoretical and Mathematical Physics, Springer, Berlin, 2008.

[17] R. C. Thompson, A determinantal inequality for positive definite matrices, Canad. Math. Bull. 4 (1961) 57-62. 
[18] X. Zhan, Matrix Inequalities, Springer, New York, 2002.

[19] X. Zhan, Matrix Theory, Graduate Studies in Mathematics, vol. 147, Amer. Math. Soc., Providence, RI, 2013.

[20] F. Zhang, Matrix Theory: Basic Results and Techniques, 2nd edition, Springer, New York, 2011.

[21] F. ZHANG, Positivity of matrices with generalized matrix functions, Acta Math. Sinica 28 (9) (2012) $1779-1786$. 